\title{
Quantitative Exploration of Opacity for Cloud Computing Systems
}

\author{
S. Naveen Krishna, Sabitha $\mathbf{R}$
}

\begin{abstract}
United cloud frameworks increment the dependability and lessen the expense of the computational help. The subsequent mix of secure private mists and less secure open mists, together with the way that assets should be situated inside various mists, emphatically influences the data stream security of the whole framework. In this paper, the mists just as elements of a combined cloud framework are alloted security levels, and a probabilistic stream delicate security model for a unified cloud framework is proposed. At that point the thought of murkiness an idea catching the security of data stream - of a distributed computing frameworks is presented, and various variations of quantitative examination of haziness are exhibited. Accordingly, one can follow the data stream in a cloud framework, and break down the effect of various asset assignment techniques by measuring the comparing murkiness attributes. Joined cloud frameworks increment the immovability and diminish the expense of the computational help. The resulting mix of secure private mists and less secure open mists, together with the way in which that advantages should be orchestrated inside various hazes, unequivocally impacts the data stream security of the whole structure. In this paper, the mists comparably as substances of a joined cloud structure are alloted security levels, and a probabilistic stream delicate security model for a united cloud framework is proposed. By then the possibility of shadowiness - an idea getting the security of data stream - of a passed on figuring frameworks is presented, and various assortments of quantitative assessment of obscurity are appeared. Thusly, one can seek after the data stream in a cloud structure, and take a gander at the effect of various asset scattering systems by surveying the differentiating dimness attributes.
\end{abstract}

Keywords: Distributed Computing, Cloud Framework, Cloud computing, opacity, Quantitative.

\section{INTRODUCTION}

The degree and significance of distributed computing is quickly expanding because of the consistently expanding interest for internet providers and interchanges. Rather than building singular data innovation framework to have databases or programming, an outsider can have these on its enormous server mists. Likewise, associations may wish to keep delicate data on their more limited servers instead of on the open ones. This has prompted the presentation of combined distributed computing wherein both open and private distributed computing assets are used.A unified cloud sends and deals with numerous distributed computing administrations, with different computational assets being assigned to various mists for both security and business concerns.

Revised Manuscript Received on December 12, 2019.

S. Naveen Krishna, Student, Saveetha School of Engineering, SIMATS, Chennai, India

Sabitha R, Professor, Saveetha School of Engineering, SIMATS, Chennai, India
Albeit a unified cloud framework can expand the unwavering quality and diminish the expense of computational help to an association, the huge number of administrations and information put away in the mists makes security hazards because of the dynamic development of information, associated gadgets, and clients between different cloud situations. Thus, it is important to track and control the data stream. So as to make such data and information detectable, one needs a conventional model depicting the data stream security inside Federated cloud frameworks. In this paper, we will present a probabilistic progress framework portrayal of the data stream in Federated cloud framework, and afterward examine security properties of the data stream utilizing darkness, which a thought catching the security of data stream.

\section{EXISTED SYSTEM}

The degree and monstrosity of circled enrolling is quickly reaching out considering the dependably developing energy for web associations and exchanges. Instead of structure singular data progression foundation to have databases or programming, an untouchable can have these on its enormous server mists.

\section{Drawbacks}

This ability and accommodation of an associated world is that its organization to purposeful aggravations. The board clients much of the time endeavor to acquire touchy information or incapacitate regular workstation capacities. Expectations much of the time include the robbery of individual or monetary information.

\section{PROPOSED SYSTEM}

Mists just as elements of a united cloud framework are relegated security levels, and a probabilistic stream touchy security model for a unified cloud framework is proposed. A distributed computing frameworks is presented, and various variations of quantitative examination of obscurity .

As it is actualized in both equipment and programming, it is most strong security protocol.It is most normal security convention utilized for wide different of uses, for example, remote correspondence, budgetary exchanges, e-business, scrambled information stockpiling and so on. 


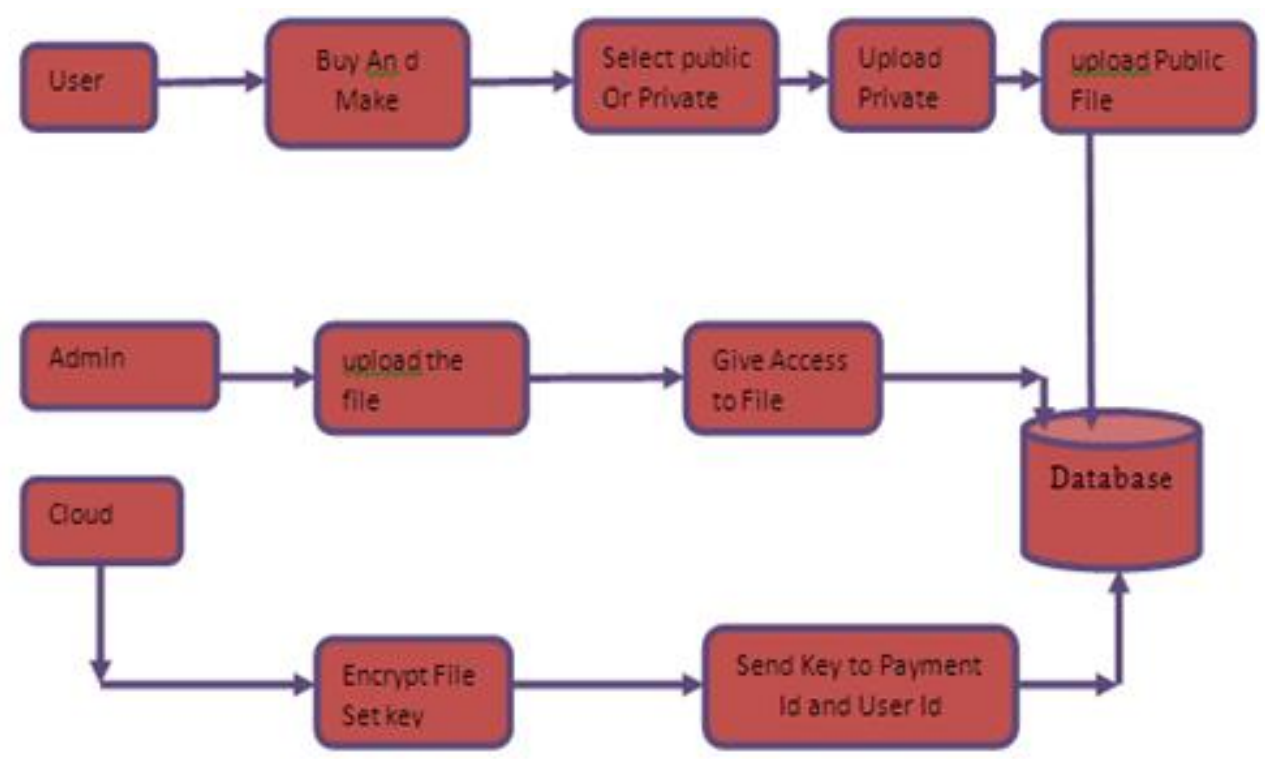

Fig. 1 System Architecture

\section{METHODOLOGY}

\section{Admin}

Authentication

Permit User to Login after Registration

User

Authentication

Register User Details

View and Select File

Payment for the File and Download

\section{RESULTS AND DISCUSSION}

The proposed system works well and shows that accuracy is the first factor to be noted. Accuracy factor is high in case of proposed system. The experimental results prove the same (Fig.2).

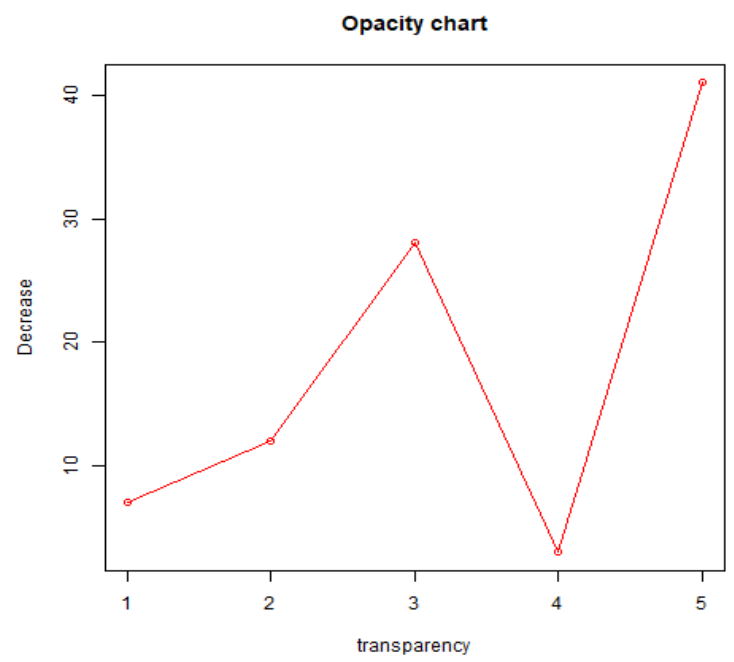

Fig. 2 Experimental Results Graph

\section{CONCLUSION}

Confirmation strategies dependent on the aftereffects of this utilizing check devices a scope of sensible contextual investigations so as to profile the value of the various thoughts of haziness talked about in this paper and position them against different methods for estimating quantitative data flow.The proposed model is the most appropriate improvement procedure to actualize for this undertaking. As it is actualized in both equipment and programming, it is most vigorous security protocol.It is most basic security convention utilized for wide different of utilizations, for example, remote correspondence, money related exchanges, e-business, scrambled information stockpiling and so forth.

\section{REFERENCES}

1. P. Watson, "A multi-level security model for partitioning work-flows over federated clouds," Journal of Cloud Computing, vol. 1,no. 1, pp. 1 $-15,2012$.

2. D. Bell and L. La Padula, "Secure computer systems: Mathematical foundations," MITRE Corporation, Tech. Rep., Mar. 1973.

3. K. Knorr, "Multilevel security and information flow in Petri net workflows," in 9th International Conference on Telecommunication Systems - Modeling and Analysis, Special Session on Security Aspects of Telecommunication Systems, 2001.

4. V. Varadharajan, "Hook-up property for information flow secure nets," in Computer Security Foundations Workshop IV, 1991. Proceedings, 1991 , pp. $154-175$.

5. K. Juszczyszyn, "Verifying enterprise's mandatory access control policies with coloured Petri nets," in Enabling Technologies: Infrastructure for Collaborative Enterprises, 2003. WET ICE 2003.Proceedings. Twelfth IEEE International Workshops on, 2003, pp. 184-189.

6. D. E. Bell, "Concerning 'modeling' of computer security," in Proceedings. 1988 IEEE Symposium on Security and Privacy, 1988,pp $8-13$.

7. H. Hiden, S. Woodman, and P. Watson, "A framework for dynamically generating predictive models of workflow execution," in Proceedings of WORKS 2013: 8th Workshop On Workflows in Support of LargeScale Science, Held in conjunction with SC13, Denver, CO,USA, November 17, 2013, 2013, pp. $77-87$.

8. J. Cala, H. Hiden, S. Woodman, and P. Watson, "Cloud computing for fast prediction of chemical activity," Future Generation Computer Systems, vol. 29, no. 7, pp. 1860 - 1869, 2013.

9. S. Woodman, H. Hiden, and P. Watson, "Applications of provenance in performance prediction and data storage optimisation," Future Generation Computer Systems, vol. 75, pp. 299 - 309, 2017. 
10. S. Sharif, P. Watson, J. Taheri, S. Nepal, and A. Y. Zomaya, "Privacyaware scheduling saas in high performance computing environments," IEEE Transactions on Parallel and Distributed Systems, vol. 28, no. 4 pp. $1176-1188,2017$.

11. J. Landauer and T. Redmond, "A lattice of information," in Computer Security Foundations Workshop VI, 1993. Proceedings, Jun 1993, pp. $65-70$.

12. J. K. Millen, "Covert channel capacity," in IEEE Symposium on Security and Privacy, 1987, pp. $60-66$.

13. Hussain A., Mkpojiogu E.O.C., Kamal F.M. (2016). Mobile video streaming applications: A systematic review of test metrics in usability evaluation. Journal of Telecommunication, Electronic and Computer Engineering. Vol 8 Issue 10. Page 35-39

14. A. McIver and C. Morgan, A probabilistic approach to information hiding. New York: Springer New York, 2003, pp. $441-460$. 